\title{
DHHFL-MABAC Approach based on DHHFLDM- LCME for Multi-criteria Group Decision Making Involving Sewage Treatment Company Selection
}

\section{Sidong Xian ( $\nabla$ sidx@163.com )}

Chongqing University of Posts and Telecommunications https://orcid.org/0000-0002-9871-4444

\section{Zhaoyu Yan}

Chongqing University of Posts and Telecommunications

\section{Wenhua Wan}

Chongqing University of Posts and Telecommunications

\section{Research Article}

Keywords: DHHFLDM-MABAC, double hierarchy hesitant fuzzy linguistic, DHHFLDM-LCME, binary contrast method, comprehensive weight

Posted Date: January 13th, 2022

DOI: https://doi.org/10.21203/rs.3.rs-1100071/v1

License: (c) (i) This work is licensed under a Creative Commons Attribution 4.0 International License. Read Full License 


\title{
DHHFL-MABAC approach based on DHHFLDM-LCME for multi-criteria group decision making involving sewage treatment company selection
}

\author{
Sidong Xian ${ }^{\mathrm{a}, \mathrm{b}, *}$, Zhaoyu Yan ${ }^{\mathrm{b}}$, Wenhua Wan ${ }^{\mathrm{c}}$ \\ ${ }^{a}$ Key Laboratory of Intelligent Analysis and Decision on Complex Systems, Chongqing University of Posts and \\ Telecommunications, Chongqing, 400065, P. R. China \\ ${ }^{b}$ School of Computer Science, Chongqing University of Posts and Telecommunications, Chongqing, 400065, \\ P.R.China \\ ${ }^{c}$ School of automation, Chongqing University of Posts and Telecommunications, Chongqing, 400065, P. R. China
}

\begin{abstract}
With the continuous acceleration of urban construction and development, ecological governance has become an important part of our green life. Therefore, how to select the appropriate governance company in the complex decision-making environment is very important. A double hierarchy hesitant fuzzy linguistic multi-attributive border approximation area method (DHHFL-MABAC) is proposed in this paper, which is based on distance measure and comprehensive weight. DHHFLMABAC method not only considers the potential loss, but also has simple calculation and stable results. The double hierarchy hesitant fuzzy linguistic distance measure based on least common multiple (DHHFLDM-LCME) is proposed, which reduces the loss of original information and makes the result more accurate. Binary contrast method is proposed and combined with entropy weight to obtain comprehensive weight, which makes the determined weight more reasonable. Finally, this method is applied to the case selected by the sewage treatment company and proved its effectiveness. Keywords: DHHFLDM-MABAC; double hierarchy hesitant fuzzy linguistic; DHHFLDM-LCME; binary contrast method; comprehensive weight.
\end{abstract}

\section{Introduction}

As the economy and society have progressed, the amount of decision-making information is increasing, and the uncertainty of decision-making environment and the complexity of problems are also increasing. The precise numerical and mathematical models cannot satisfy the requirement of decision-makers to describe the uncertainty and hesitation in actual decision-making. Base on this, in 1965, Zadeh first put forward the concept of fuzzy sets [1], which created a theoretical method for studying the problem of fuzziness or uncertainty. In 1975, Zadeh put forward the concept of linguistic variables 2]. Scholars conducted further research and proposed new forms of language

* Sidong Xian

Email address: sidx@163.com (Sidong Xian) 
expression. In 1967, Goguen proposed the L-fuzzy sets 3]. The interval valued fuzzy sets 4] was proposed by Tuksen in 1986, Herrera put forward the concept of 2-tuple fuzzy sets[5] in 2000. However, the above expressions of fuzzy information only enrich and expand the value range of the membership function. They cannot broaden the dimensionality of information expression. In response to the above problems, scholars have made further research on it. The intuitionistic fuzzy sets [6] and interval intuitionistic fuzzy sets [7] were obtained by further extending the fuzzy sets, both of which take into account the information of the two dimensions of hesitation and membership, which makes the expression of fuzzy information more complete.

To better figure out the problem of decision-making in a more complex environment, Torra proposed the defination of hesitant fuzzy sets(HFS) [8] in 2010. It solves the problem that decision maker is hesitant between two or finite membership values. Based on this idea, some scholars have studied and extended the hesitant fuzzy sets 9, 10. In a hesitant fuzzy linguistic environment, decision methods and models [11, 12, 13, which used to work out multi-attribute decision making(MADM), were proposed.

Soon, in order to increase the appropriacy of information expression in different decision-making situations, the double hierarchy linguistic term set(DHLTS) has been developed based on the linguistic term sets (LTS). Later, in view of the idea of HFS and DHLTS, Gou[14] put forward the concept of double hierarchy hesitant fuzzy linguistic term set (DHHFLTS). When the decisionmaker swings about different linguistic information, DHHFLTS can make the decision-maker express the evaluation more accurately. Gou[15] put forward the concept of distance and similarity measure between double hierarchy hesitant fuzzy linguistic elements(DHHFLEs) and DHHFLEs. DHHFLTS 16, 17, 18, 19, 20, was frequently applied to practical decision-making problems based on advantages of it. In MADM problems, the weight of attributes represents the relative importance of attributes. The right selection of decision-making procedures and the calculation of weight can assist decision-makers to make correct judgements and choices in a double hierarchy hesitant fuzzy linguistic (DHHFL) environment.

Therefore, determining the weight of attributes is important for ranking alternatives. Many scholars have made further research on weight calculation and decision-making methods. R. Krishankumar[21] proposed the VIKOR method based on the DHHFLTS, which ranked alternatives based on their proximity to the ideal solution. $\mathrm{Xu}$ and Zhang 22$]$ put forward the method of DHHFL-BWM-DSET, which solved the MADM problem with unknown weights under the DHHFL environment. The COPRAS method[18] was proposed by R Krishankumar under the DHHFLTS, which distinguished and compares the priorities of objects from different perspectives. Liu[23] put forward the TODIM method of the DHHFLTS for multi-criteria group decision-making method. Wang[24] proposed ORESTE method based on the DHHFLTS.

According to the above literature analysis, we can find the following challenges: 
(1) In DHHFLTS, the distance measure calculation of DHHFLEs of different lengths will have the problem of linguisitic information loss.

(2) In the method of weight calculation, which cannot effectively express the importance of multiple attributes and decision maker's preference information.

(3) The existing decision-making methods are computationally complex and cannot sort alternatives efficiently and reasonably.

We are motivated by these challenges, and this paper has made some contributions:

(1) The double hierarchy hesitant fuzzy linguistic distance measure based on least common multiple(DHHFLDM-LCME) is proposed, which can reduce and overcome the loss of linguisitic information.

(2) Binary contrast method is proposed, which can express the preference information of decisionmakers well. On this basis, combined with entropy weight to obtain comprehensive weight.

(3) DHHFL-MABAC method based on DHHFLDM-LCME and comprehensive weight is proposed, the reasonability and effectiveness of this method are illustrated by the cases selected by the sewage treatment company.

The other sections of the paper are as below: In Section 2, the definations of DHLTS and DHHFLTS are reviewed. In Section 3, the DHHFLDM-LCME is proposed. Section 4 proposes the DHHFL-MABAC method based on DHHFLDM-LCME and comprehensive weight. Section 5 introduces a case study on the selection of sewage treatment companies. Section 6 conducts a sensitivity analysis. In Section 7, a summary of this paper is given.

\section{Preliminaries}

In this part, we have made some preparations for the whole work.

\subsection{Double hierarchy linguistic term set}

During the decision-making process, to handle ambiguity and uncertainty, some scholars have proposed the HFLTS, which has made a breakthrough, and conducted in-depth research on decisionmaking and future directions [25, 26]. However, considering that the vocabulary we use to describe information in our daily life is becoming more and more abundant, Gou[14] put forward the concept of DHLTS and DHHFLTS, they are able to articulate more detailed linguistic information, which corresponds to people's perceptions. The following is the concept of DHLTS.

Definition 1. 14] Let $S=\left\{s_{t} \mid t=-\tau, \cdots,-1,0,1, \cdots, \tau\right\}$ be the first hierarchy LTS and $O=$ $\left\{o_{k} \mid k=-\varsigma, \cdots,-1,0,-1, \cdots, \varsigma\right\}$ be the second hierarchy LTS, which are completely independent of each other. The DHLTS $S_{O}$ has the following mathematical expression::

$$
S_{O}=\left\{s_{t<o_{k}}>t=-\tau, \cdots,-1,0,1, \cdots, \tau ; k=-\varsigma, \cdots,-1,0,-1, \cdots, \varsigma\right\}
$$


When the first hierarchy linguistic term is $s_{t}, o_{k}$ denotes the second hierarchy linguistic term. We refer $s_{t<o_{k}>}$ as the double hierarchy linguistic term (DHLT),

Remark 1. There are four cases according to different values of $t[19]$ :

If $t<0$, the first hierarchy LTS $S=\left\{s_{t} \mid t<0\right\}$ has a negative connotation, and the second hierarchy LTS must be picked in decreasing order.

If $t>=0$, the first hierarchy LTS $S=\left\{s_{t} \mid t<0\right\}$ has a non-negative connotation, and the second hierarchy LTS must be picked in ascending order.

if $t=-\tau$, then we only care about the first half of the second hierarchy of LTS, $O=\left\{o_{k} \mid k=\right.$ $0,1, \cdots, \varsigma\}$.

if $t=\tau$, then we only care about the second hierarchy of LTS, $O=\left\{o_{k} \mid k=-\tau, \cdots,-1,0\right\}$.

\subsection{Double hierarchy hesitant fuzzy linguistic term set}

During the decision-making process, there are complex linguistic terms that cannot be clearly articulated in a single linguistic term, for example "between a little high and very high". Therefore, Gou [14] defined the concept of DHHFLTS based on DHLTS. DHHFLTS provide decision makers a richer and more flexible environment to express complex linguistic information. The following are some fundamental DHHFLTS informations.

Definition 2. 14 Let $\mathrm{X}$ be a fixed set, $S_{O}$ be a double hierarchy LTS, A double hierarchy hesitant fuzzy linguistic term set on $X, H_{S_{o}}$ is expressed in terms of a membership function returning a subset of $S_{o}$ when applied to $X$, and is expressed mathematically:

$$
H_{s_{o}}=\left\{<x_{i}, h_{s_{o}}\left(x_{i}\right)>\mid x_{i} \in X\right\}
$$

In which $\mathrm{h}_{s_{o}}\left(x_{i}\right)$ is a set of values from $S_{o}$ that represents the possible degree of membership of element $x_{i} \in X$ to the set $H_{s_{o}}$ is:

$$
\begin{array}{r}
h_{s_{o}}\left(x_{i}\right)=\left\{s_{\phi_{l}<o_{\varphi_{l}}}>\left(x_{i}\right) \mid s_{\phi_{l}<o_{\varphi_{l}}}>\in S_{o} ; l=1,2,3 \cdots, L ; \phi_{l}=-\tau, \cdots,\right. \\
\left.-1,0,1, \cdots, \tau ; \varphi_{l}=-\varsigma, \cdots,-1,0,1, \cdots, \varsigma\right\}
\end{array}
$$

with $L$ being the number of DHLTs in $h_{s_{o}}\left(x_{i}\right)$ and $s_{\phi_{l}<o_{\varphi_{l}}>}(l=1,2,3, \cdots, L)$ in each $h_{s_{o}}\left(x_{i}\right)$ being the continuous terms in $S_{o} . h_{s_{o}}\left(x_{i}\right)$ is called the double hierarchy hesitant fuzzy linguistic element(DHHFLE), which stands for the possible degree of the linguistic varible $x_{i}$ to $S_{o}$. Next, in order to better understand DHHFLTS, we give an example.

Example 1. Suppose $S=\left\{s_{-2}=\right.$ null, $s_{-1}=$ small, $s_{0}=$ medium, $s_{1}=$ big, $s_{2}=$ huge $\}$ and $O=\left\{o_{-2}=\right.$ slightly, $o_{-1}=$ a little,$o_{0}=$ just right, $o_{1}=$ very,$o_{2}=$ entirely $\}$ are the first hierarchy and the second hierarchy LTSs, when we describe some complicated linguistic terms, for example, "just right medium " and "between a little small and entirely big", we can use DHHFLEs $\left\{s_{0<o_{o}>}\right\}$ and $\left\{s_{0<o_{o}>}, s_{1<o_{3}>}\right\}$. 
Definition 3. 15] Let $S_{O}$ be a DHLTS and $h_{s_{o}}\left(x_{i}\right)=\left\{s_{\phi_{l}<o_{\varphi_{l}}>}\left(x_{i}\right) \mid s_{\phi_{l}<o_{\varphi_{l}}}>\in S_{o} ; l=1,2,3 \cdots, L ; l=\right.$ $\left.1,2, \cdots, \# h_{s_{o}}\right\}\left(\# h_{s_{o}}\right.$ represents the number of $h_{s_{o}}$ in DHLTS) be a DHHFLE, after that we can define:

$$
v\left(h_{s_{o}}\left(x_{i}\right)\right)=1-\frac{1}{\# h_{s_{o}}}
$$

the hesitant degree of the DHHFLE $h_{s_{o}}\left(x_{i}\right)$. The degree of hesitation is a very important characteristic of DHHFLE, which reflects the degree of hesitation of the decision-maker. The quality of DHHFLE will become lower as the degree of hesitation increases.

In order to simplify the operation of DHHFLTS, two equivalent conversion functions are developed to convert DHFLT to fuzzy numbers, as shown below:

Definition 4. 14] Let $\overline{S_{o}}=\left\{s_{t<o_{k}>} \mid t \in[-\tau, \tau] ; k \in[-\varsigma, \varsigma]\right\}$ be a virtual DHLTS, $S_{O}$ be a DHLTS, $h_{s_{o}}\left(x_{i}\right)$ be a DHHFLE with $L$ being the number of linguistic terms in $h_{s_{o}}$, and $h_{\gamma}=\left\{\gamma_{l} \mid \gamma_{l} \in\right.$ $[0,1] ; l=1,2,3, \cdots, L\}$ be a set of numerical scales. Then the numerical scale $\gamma_{l}$ and the subscript $\left(\phi_{l}, \varphi_{l}\right)$ of the DHLT $s_{\phi_{l<\varphi_{l}}>}$ that express the equivalent information to the numerical scale $\gamma_{l}$ can be transformed to each other by two function $f$ and $f^{-1}$ :

$$
\begin{gathered}
f:[-\tau, \tau] \times[-\varsigma, \varsigma] \rightarrow[0,1], f\left(\phi_{l}, \varphi_{l}\right)=\frac{\varphi_{l}+\varsigma\left(\tau+\phi_{l}\right)}{2 \tau \varsigma}=\gamma_{l} \\
f^{-1}:[0,1] \rightarrow[-\tau, \tau] \times[-\varsigma, \varsigma], \\
f^{-1}\left(\gamma_{l}\right)=\left[2 \tau \gamma_{l}-\tau\right]<o_{\varsigma\left(2 \tau \gamma_{l}-\tau-\left[2 \tau \gamma_{l}-\tau\right]\right)}>=\left[2 \tau \gamma_{l}-\tau\right]+1<o_{\varsigma\left(\left(2 \tau \gamma_{l}-\tau-\left[2 \tau \gamma_{l}-\tau\right]\right)-1\right)}>
\end{gathered}
$$

Let $\Phi \times \Psi$ as the set of all DHHFLEs on $\overline{S_{o}}, \Omega$ be the set of all numerical scales. Then the conversion function $F$ and $F^{-1}$ between $h_{s_{o}}$ and $h_{\gamma}$ based on $f$ and $f^{-1}$ are:

$$
\begin{aligned}
& F: \Phi \times \Psi \rightarrow \Omega, \\
& F\left(h_{s_{o}}\right)=F\left(\left\{s_{\phi_{l}<o_{\varphi_{l}}>} \mid s_{\phi_{l}<o_{\varphi_{l}}}>\in \overline{S_{o}} ; l=1,2, \cdots, L ; \phi_{l} \in[-\tau, \tau] ; \varphi_{l} \in[-\varsigma, \varsigma]\right\}\right) \\
& =\left\{\gamma_{l} \mid \gamma_{l}=f\left(\phi_{l}, \varphi_{l}\right) ; l=1,2, \cdots, L\right\}=h_{\gamma} \\
& F^{-1}: \Omega \rightarrow \Phi \times \Psi \\
& F^{-1}\left(h_{\gamma}\right)=F^{-1}\left(\left\{\gamma_{l} \mid \gamma_{l} \in[0,1] ; l=1,2, \cdots, L\right\}\right)=\left\{s_{\phi_{l}<o_{\varphi_{l}}}>\mid \phi_{l}<o_{\varphi_{l}}>=f^{-1}\left(\gamma_{l}\right)\right\}=h_{s_{o}}
\end{aligned}
$$

Definition 5. 14 Let $S_{O}$ be a DHLTS, $h_{s_{o}}$ be a DHHFLE, $L$ is the length of $h_{s_{o}}$. The following is the definition of the expected value of $h_{s_{o}}$.

$$
E\left(h_{s_{o}}\right)=\frac{1}{L} \sum_{l=1}^{L} F\left(s_{\phi_{l}<o_{\varphi_{l}}}>\right)
$$

In different DHHFLTS, the quantity of linguistic terms may be different. Therefore, it is necessary to standardize the DHHFLTSs so that they contain the same quantity of linguistic terms. 
To ensure the integrity of the DHHFLTS information before and after expansion, this paper gives the double hierarchy hesitant fuzzy linguistic least common multiple expansion method, which is as follows:

Definition 6. Let $h_{s_{o}}=\left\{s_{\phi_{0}<o_{\varphi_{0}}}>, s_{\phi_{1}<o_{\varphi_{2}}}>, \cdots, s_{\phi_{i}<o_{\varphi_{j}}}>\right\}$ be a DHHFLE with $L$ linguistic term on $S_{o}$, Namely, $h_{s_{o}} \in \operatorname{DHHFLTS}\left(S_{o}\right)$. Then $H_{s_{o}}$ is the set after expansion with $h_{s_{o}}$, which is defined as follows:

$H_{s_{o}}=\{\underbrace{s_{\phi_{0}<o_{\varphi_{0}}>}, s_{\phi_{0}<o_{\varphi_{0}}>}, s_{\phi_{0}<o_{\varphi_{0}}}>}_{\text {times }}, \underbrace{s_{\phi_{1}<o_{\varphi_{2}}>}, s_{\phi_{1}<o_{\varphi_{2}}>}, s_{\phi_{1}<o_{\varphi_{2}}>}}_{\text {times }} \cdots, \underbrace{s_{\phi_{i}<o_{\varphi_{j}}}>, s_{\phi_{i}<o_{\varphi_{j}}>}, s_{\phi_{i}<o_{\varphi_{j}}}>}_{t \text { times }}\}$

The $t$ represents the multiplicity of each element $s_{k}$ of $h_{s_{o}}$ in $H_{s_{o}}$.

\section{The double hierarchy hesitant fuzzy linguistic distance measure based on least com- mon multiple}

The existing literature defines a lot of distance measure methods, but most of these distance measure assume that the length of the DHHFLTSs is the same, or use the $\alpha$ criterion and $\beta$ criterion to subjectively normalize the DHHFLEs of different lengths. However, these distance measures have certain shortcomings:

(1) In actual decision-making problems, few different decision makers give DHHFLTS of the same length.

(2) $\alpha$ and $\beta$ criteria will destroy hesitation the completeness of the information before and after the expansion of the fuzzy linguistic term set.

Hence based on the idea of normalization method in literature 28], DHHFLDM-LCME is proposed in this paper.

Definition 7. Let $h_{s_{o}}^{1}$ and $h_{s_{o}}^{2}$ are two DHHFLEs of length $L_{1}$ and $L_{2}$ respectively. The following is a mathematical representation of DHHFLDM-LCME.

$$
d_{E}\left(h_{s_{o}}^{1}, h_{s_{o}}^{2}\right)=\frac{1}{L C M} \sum_{\mathrm{k}=1}^{L C M}\left|F\left(H_{k}^{1}\right)-F\left(H_{k}^{2}\right)\right|
$$

In which LCM is the least common multiple of $L_{1}$ and $L_{2}$, and $F$ is a monotone function, $H_{k}$ is the k-th element in the $H_{s_{o}}$ set after is $h_{s_{o}}$ expanded.

Theorem Let $\left(h_{s_{o}}^{1}, h_{s_{o}}^{2}\right)$ are two DHHFLEs, then:

(1) Boundary: $0 \leq d_{E}\left(h_{s_{o}}^{1}, h_{s_{o}}^{2}\right) \leq 1$;

(2) Symmetry: $d_{E}\left(h_{s_{o}}^{1}, h_{s_{o}}^{2}\right)=d_{E}\left(h_{s_{o}}^{2}, h_{s_{o}}^{1}\right)$;

(3) Reflexivity: $d_{E}\left(h_{s_{o}}^{1}, h_{s_{o}}^{1}\right)=0$;

(4) Inequality: $d_{E}\left(h_{s_{o}}^{1}, h_{s_{o}}^{2}\right)+d_{E}\left(h_{s_{o}}^{2}, h_{s_{o}}^{3}\right) \geq d_{E}\left(h_{s_{o}}^{1}, h_{s_{o}}^{3}\right)$. 
Where (1), (2) and (3) are obvious, we will not prove it here. The proof of Theorem (4) will be given below:

Proof:

We will classify and discuss from two major aspects: when the lengths of the DHHFLEs are the same, we can get the following situations:

if $h_{s_{o}}^{1}=h_{s_{o}}^{2}=h_{s_{o}}^{3}$, according to Theorem (3), there are:

$d_{E}\left(h_{s_{o}}^{1}, h_{s_{o}}^{2}\right)=d_{E}\left(h_{s_{o}}^{2}, h_{s_{o}}^{3}\right)=d_{E}\left(h_{s_{o}}^{1}, h_{s_{o}}^{3}\right)=0, d_{E}\left(h_{s_{o}}^{1}, h_{s_{o}}^{2}\right)+d_{E}\left(h_{s_{o}}^{2}, h_{s_{o}}^{3}\right) \geq d_{E}\left(h_{s_{o}}^{1}, h_{s_{o}}^{3}\right)$

Apparently established;

if $h_{s_{o}}^{1}=h_{s_{o}}^{2} \neq h_{s_{o}}^{3}$, according to Theorem (3), there are:

$d_{E}\left(h_{s_{o}}^{1}, h_{s_{o}}^{2}\right)+d_{E}\left(h_{s_{o}}^{2}, h_{s_{o}}^{3}\right)=d_{E}\left(h_{s_{o}}^{2}, h_{s_{o}}^{3}\right), d_{E}\left(h_{s_{o}}^{2}, h_{s_{o}}^{3}\right)=d_{E}\left(h_{s_{o}}^{1}, h_{s_{o}}^{3}\right)$,

$d_{E}\left(h_{s_{o}}^{1}, h_{s_{o}}^{2}\right)+d_{E}\left(h_{s_{o}}^{2}, h_{s_{o}}^{3}\right)=d_{E}\left(h_{s_{o}}^{1}, h_{s_{o}}^{3}\right)$.

In the same way, it can also be proved under the two conditions of $h_{s_{o}}^{1} \neq h_{s_{o}}^{2}=h_{s_{o}}^{3}$ and $h_{s_{o}}^{1}=h_{s_{o}}^{3} \neq$ $h_{s_{o}}^{2}$.

When the lengths of the DHHFLEs are different, we set the lengths of $h_{s_{o}}^{1}, h_{s_{o}}^{2}$, and $h_{s_{o}}^{3}$ to be $L_{1}$, $L_{2}$, and $L_{3}$ respectively, then $a, b$ and $c$ are the least common multiples between them respectively, and the least common multiple of the three of them is $n$, according to Definition 7, there are:

$$
\begin{aligned}
& d_{E}\left(h_{s_{o}}^{1}, h_{s_{o}}^{2}\right)=\frac{1}{a} \sum_{k=1}^{a}\left|F\left(H_{k}^{1}\right)-F\left(H_{k}^{2}\right)\right| \\
& d_{E}\left(h_{s_{o}}^{2}, h_{s_{o}}^{3}\right)=\frac{1}{b} \sum_{k=1}^{b}\left|F\left(H_{k}^{2}\right)-F\left(H_{k}^{3}\right)\right| \\
& d_{E}\left(h_{s_{o}}^{1}, h_{s_{o}}^{3}\right)=\frac{1}{c} \sum_{k=1}^{c}\left|F\left(H_{k}^{1}\right)-F\left(H_{k}^{3}\right)\right| \\
& d_{E}\left(h_{s_{o}}^{1}, h_{s_{o}}^{2}\right)=\frac{1}{a} \sum_{k=1}^{a}\left|F\left(H_{k}^{1}\right)-F\left(H_{k}^{2}\right)\right|=\frac{1}{a k_{1}} k_{1} \sum_{l=1}^{a}\left|F\left(H_{k}^{1}\right)-F\left(H_{k}^{2}\right)\right| \text { which } k_{1}, k_{2}, k_{3} \text { are con- }
\end{aligned}
$$
stants respectively, make $a k_{1}=n, a k_{2}=n, a k_{3}=n$. Thoughts based on the principle of least common multiple, according to Definition 6 , there are:

$$
\frac{1}{a k_{1}} k_{1} \sum_{l=1}^{a}\left|F\left(H_{k}^{1}\right)-F\left(H_{k}^{2}\right)\right|=\frac{1}{a k_{1}} \sum_{l=1}^{a k_{1}}\left|F\left(H_{k}^{1}\right)-F\left(H_{k}^{2}\right)\right|=\frac{1}{n} \sum_{l=1}^{n}\left|F\left(H_{k}^{1}\right)-F\left(H_{k}^{2}\right)\right|
$$

In the same way, there are:

$$
\begin{aligned}
& d_{E}\left(h_{s_{o}}^{2}, h_{s_{o}}^{3}\right)=\frac{1}{n} \sum_{k=1}^{n}\left|F\left(H_{k}^{2}\right)-F\left(H_{k}^{3}\right)\right| \\
& d_{E}\left(h_{s_{o}}^{1}, h_{s_{o}}^{3}\right)=\frac{1}{n} \sum_{k=1}^{n}\left|F\left(H_{k}^{1}\right)-F\left(H_{k}^{3}\right)\right|
\end{aligned}
$$

If we want to prove $d_{E}\left(h_{s_{o}}^{1}, h_{s_{o}}^{2}\right)+d_{E}\left(h_{s_{o}}^{2}, h_{s_{o}}^{3}\right) \geq d_{E}\left(h_{s_{o}}^{1}, h_{s_{o}}^{3}\right)$, we only need to prove:

$\frac{1}{n} \sum_{k=1}^{n}\left|F\left(H_{k}^{1}\right)-F\left(H_{K}^{2}\right)\right|+\frac{1}{n} \sum_{k=1}^{n}\left|F\left(H_{k}^{2}\right)-F\left(H_{k}^{3}\right)\right| \geq \frac{1}{n} \sum_{k=1}^{n}\left|F\left(H_{k}^{1}\right)-F\left(H_{k}^{3}\right)\right|$, and that,

$\frac{1}{n} \sum_{k=1}^{n}\left(\left|F\left(H_{k}^{1}\right)-F\left(H_{k}^{2}\right)\right|+\left|F\left(H_{k}^{2}\right)-F\left(H_{k}^{3}\right)\right|-\left|F\left(H_{k}^{1}\right)-F\left(H_{k}^{3}\right)\right|\right) \geq 0$

we can get the following six situations:

if $F\left(H_{k}^{1}\right)>F\left(H_{k}^{2}\right)>F\left(H_{k}^{3}\right)$, there are:

$\frac{1}{n} \sum_{k=1}^{n}\left(F\left(H_{k}^{1}\right)-F\left(H_{k}^{2}\right)+F\left(H_{k}^{2}\right)-F\left(H_{k}^{3}\right)-\left(F\left(H_{k}^{1}\right)-F\left(H_{k}^{3}\right)\right)\right)$

$=\frac{1}{n} \sum_{k=1}^{n}\left(F\left(H_{k}^{1}\right)-F\left(H_{k}^{3}\right)-F\left(H_{k}^{1}\right)+F\left(H_{k}^{3}\right)\right)=0$

if $F\left(H_{k}^{1}\right)>F\left(H_{k}^{3}\right)>F\left(H_{k}^{2}\right)$, there are: 


$$
\begin{aligned}
& \frac{1}{n} \sum_{k=1}^{n}\left(F\left(H_{k}^{1}\right)-F\left(H_{k}^{2}\right)+F\left(H_{k}^{3}\right)-F\left(H_{k}^{2}\right)-\left(F\left(H_{k}^{1}\right)-F\left(H_{k}^{3}\right)\right)\right) \\
& =\frac{1}{n} \sum_{k=1}^{n}\left(2 F\left(H_{k}^{3}\right)-2 F\left(H_{k}^{2}\right)\right)>0
\end{aligned}
$$

if $F\left(H_{k}^{2}\right)>F\left(H_{k}^{1}\right)>F\left(H_{k}^{3}\right)$, there are:

$\frac{1}{n} \sum_{k=1}^{n}\left(F\left(H_{k}^{2}\right)-F\left(H_{k}^{1}\right)+F\left(H_{k}^{2}\right)-F\left(H_{k}^{3}\right)-\left(F\left(H_{k}^{1}\right)-F\left(H_{k}^{3}\right)\right)\right)$

$=\frac{1}{n} \sum_{k=1}^{n}\left(2 F\left(H_{k}^{2}\right)-2 F\left(H_{k}^{1}\right)\right)>0$

if $F\left(H_{k}^{2}\right)>F\left(H_{k}^{3}\right)>F\left(H_{k}^{1}\right)$, there are:

$\frac{1}{n} \sum_{k=1}^{n}\left(F\left(H_{k}^{2}\right)-F\left(H_{k}^{1}\right)+F\left(H_{k}^{2}\right)-F\left(H_{k}^{3}\right)-\left(F\left(H_{k}^{3}\right)-F\left(H_{k}^{1}\right)\right)\right)$

$=\frac{1}{n} \sum_{k=1}^{n}\left(2 F\left(H_{k}^{2}\right)-2 F\left(H_{k}^{3}\right)\right)>0$

if $F\left(H_{k}^{3}\right)>F\left(H_{k}^{1}\right)>F\left(H_{k}^{2}\right)$, there are:

$\frac{1}{n} \sum_{k=1}^{n}\left(F\left(H_{k}^{1}\right)-F\left(H_{k}^{2}\right)+F\left(H_{k}^{3}\right)-F\left(H_{k}^{2}\right)-\left(F\left(H_{k}^{3}\right)-F\left(H_{k}^{1}\right)\right)\right)$

$=\frac{1}{n} \sum_{k=1}^{n}\left(2 F\left(H_{k}^{1}\right)-2 F\left(H_{k}^{2}\right)\right)>0$

if $F\left(H_{k}^{3}\right)>F\left(H_{k}^{2}\right)>F\left(H_{k}^{1}\right)$, there are:

$\frac{1}{n} \sum_{k=1}^{n}\left(F\left(H_{k}^{2}\right)-F\left(H_{k}^{1}\right)+F\left(H_{k}^{3}\right)-F\left(H_{k}^{2}\right)-\left(F\left(H_{k}^{3}\right)-F\left(H_{k}^{1}\right)\right)\right)$

$=0$

In summary, the proof of Theorem.(4) is complete.

Example 2. Let $S_{o}=\left\{s_{-2}=\right.$ none, $s_{-1}=$ small, $s_{0}=$ medium, $s_{1}=$ big, $s_{2}=$ huge $; o_{-2}=$ slightly, $o_{-1}=$ a little,$o_{0}=$ just right, $o_{1}=$ very, $o_{2}=$ entirely $\}$ is a DHHFLTS, $h_{s_{o}}^{1}=\left\{s_{-1<o_{1}>}, s_{0<o_{1}>}, s_{1<o_{2}>}\right\}$ and $h_{s_{o}}^{2}=\left\{s_{1<o_{1}>}, s_{2<o_{0}>}\right\}$ are two DHHFLEs, Further, based on the least common multiple expansion rules and standardized treatment are:

$$
\begin{aligned}
& H_{s_{o}}^{1}=\left\{s_{-1<o_{1}>}, s_{-1<o_{1}>}, s_{0<o_{1}>}, s_{0<o_{1}>}, s_{1<o_{2}>}, s_{1<o_{2}>}\right\}, \\
& H_{s_{o}}^{2}=\left\{s_{1<o_{-1}>}, s_{1<o_{-1}>}, s_{1<o_{-1}>}, s_{1<o_{1}>}, s_{1<o_{1}>}, s_{1<o_{1}>}\right\} .
\end{aligned}
$$

Then we can use the above transformation equation(7) to get the new set:

$$
\begin{aligned}
& F\left(H_{s_{o}}^{1}\right)=\{0.375,0.375,0.625,0.625,1,1\} . \\
& F\left(H_{s_{o}}^{2}\right)=\{0.625,0.625,0.625,0.875,0.875,0.875\} .
\end{aligned}
$$

And then according to the Eq.(11), we can figure out the distance between $h_{s_{o}}^{1}$ and $h_{s_{o}}^{2}$ :

$$
d_{E}\left(h_{s_{o}}^{1}, h_{s_{o}}^{2}\right)=\frac{1}{L C M} \sum_{\mathrm{k}=1}^{L C M}\left|F\left(H_{k}^{1}\right)-F\left(H_{k}^{2}\right)\right|=0.167 \text {. }
$$

\section{The DHHFL-MABAC method based on DHHFLDM-LCME and comprehensive weight}

Since DHHFLDM-LCME and comprehensive weight are prepared for DHHFL-MABAC method, so we first introduce the comprehensive weight based on binary contrast method and entropy weight, and then introduce the calculation steps of DHHFL-MABAC method in detail.

The comprehensive weighted method combines the entropy weight method 29] 30] for calculating objective attribute weights with the binary contrast mothed for calculating subjective attribute 
weight. It effectively overcomes the shortcomings of subjective attribute weight and objective attribute weight.

\subsection{Binary contrast method}

The described binary contrast method uses the empirical knowledge of decision makers to determine the subjective weight of attributes. Suppose the attribute set is $E=\left\{e_{1}, e_{2}, \cdots, e_{n}\right\}$.

Step 1. Compare the superiority of adjacent attributes according to the constraints of people's life experience and environmental factors. If the first one has a higher degree of superiority than the second one, swap the two of them.

Step 2. Do the same work for each pair of adjacent attributes, from the first pair at the beginning to the last pair at the end. At this point, the last attribute should be the attribute with the highest degree of superiority.

Step 3. Repeat the above steps for all attributes except the last one.

Step 4. Repeat the above steps for fewer and fewer attributes until there is no pair of attributes to compare. We can get an ascending set of attributes sorted by superiority.

Step 5. According to the sorted set of attributes and weighting function, we can get the subjective weight of each attribute, $w_{j}^{1}=\left\{w_{1}^{1}, w_{2}^{1}, \cdots, w_{n}^{1}\right\}$.

$$
w_{i}^{1}=\frac{1}{2^{i-1}} \times \frac{2^{n-1}}{2^{n}-1}, i=1,2, \cdots n
$$

Where $n$ is the total quantity of attributes, $\sum_{i=1}^{n} w_{i}^{1}=1,0 \leq w_{i}^{1} \leq 1$.

\subsection{Entropy weight based on DHHFLTS}

The entropy weight method 30] calculates the weight of each attribute, and then evaluates each attribute based on its weight. In the DHHFL environment, the following steps are taken to calculate the weight through the entropy weight method.

Step 1. Obtain the decision information which take the form of DHHFLTS, and construct the evaluation matrix $R$.

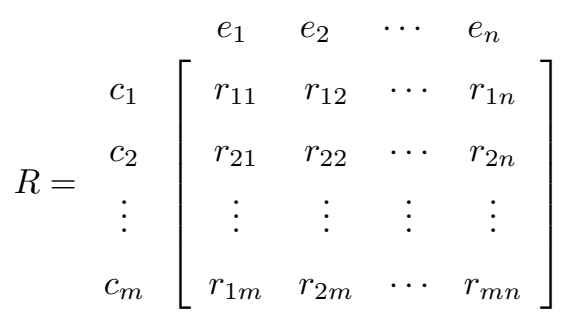

Step 2. Normalize evaluation matrix $R$ into $R^{1}$ by Eq.(14).

$$
r_{i j}^{1}=\frac{r_{i j}-\min \left(r_{j}\right)}{\max \left(r_{j}\right)-\min \left(r_{j}\right)}, \quad i=1,2, \cdots, m ; j=1,2, \cdots n
$$




$$
R^{1}=\left[\begin{array}{cccc}
r_{11}^{1} & r_{12}^{1} & \cdots & r_{1 n}^{1} \\
r_{21}^{1} & r_{22}^{1} & \cdots & r_{2 n}^{1} \\
\vdots & \vdots & \cdots & \vdots \\
r_{m 1}^{1} & r_{m 2}^{1} & \cdots & r_{m n}^{1}
\end{array}\right]
$$

In which $r_{i j}^{1}$ stands for the evaluation value of the $\mathrm{j}$-th attribute under the $\mathrm{i}$-th alternative after standardization in the decision matrix, the $\max \left(r_{j}\right)$ and $\min \left(r_{j}\right)$ are the maximum and minimum values of the $\mathrm{j}$-th attribute, respectively.

Step 3. According to the following formula, we can get the information entropy of each attribute.

$$
\begin{gathered}
E_{j}=-\frac{1}{\ln m} \sum_{i=1}^{m} P_{i j} \ln P_{i j}, \quad j=1,2, \cdots n ; i=1,2, \cdots, m . \\
P_{i j}=\frac{r_{i j}^{1}}{\sum_{i=1}^{m} r_{i j}^{1}}
\end{gathered}
$$

Step 4. Each attribute's weight value is calculated using formula as follows.

$$
w_{j}^{2}=\frac{1-E_{j}}{\sum_{j=1}^{n}\left(1-E_{j}\right)}
$$

\subsection{The comprehensive weight}

To compensate for the shortcomings of subjective and objective weights, the decision maker's preference for attributes and the importance of attributes themselves are considered, we combine binary contrast method and entropy weight to obtain comprehensive weights, so as to make the decision results true and reliable.

Suppose the subjective attribute weight obtained by the binary contrast method is $w_{j}^{1}=$ $\left\{w_{1}^{1}, w_{2}^{1}, \cdots, w_{n}^{1}\right\}$, where $\sum_{i=1}^{n} w_{i}^{1}=1,0 \leq w_{i}^{1} \leq 1$, according to the entropy weight method, the objective attribute weight is $w_{j}^{2}=\left\{w_{1}^{2}, w_{2}^{2}, \cdots, w_{n}^{2}\right\}$, where $\sum_{i=1}^{n} w_{i}^{2}=1,0 \leq w_{i}^{2} \leq 1$. According to the calculated $w_{j}^{1}$ and $w_{j}^{2}$, we can get the comprehensive weight of attributes:

$$
w_{j}=\lambda w_{j}^{1}+(1-\lambda) w_{j}^{2}, j=1,2, \cdots n .
$$

Where $\lambda$ is a parameter, $\sum_{i=1}^{j} w_{\mathrm{i}}=1,0 \leq w_{i} \leq 1$. When the decision maker has a preference for subjective weight and objective weight, $\lambda$ changes with the decision maker's preference information.

The comprehensive weight is obtained by the linear combination of objective weight and subjective weight. According to additive effect, the value of comprehensive weight is positively correlated with the value of subjective and objective weight. Moreover, the comprehensive weights overcome the limitation of considering only the influence of subjective or objective factors. 


\subsection{The DHHFL-MABAC method based on DHHFLDM-LCME and comprehensive weight}

MABAC method 32] is a MADM method. The operation method is simple and practical. The MABAC method's 31] basic idea is that to computer the border approximation area $(B A A)$ of each attribute first. Then the distance between the $B A A$ and each attribute is calculated. Finally, calculate the global distance of each alternative.

The classic MABAC method [33, 34] ranks the alternatives by calculating the global distance. When calculating the distance, there will be a problem of information loss, resulting in inaccurate sorting results. In complex decision-making problems, reasonable attribute weights are not considered. Based on this, this paper put forward a DHHFL-MABAC method based on DHHFLDMLCME and comprehensive weight to solve the MADM problem.

It is assumed that the alternative set is $C=\left\{c_{1}, c_{2}, \cdots, c_{n}\right\}$, the attribute set is $E=\left\{e_{1}, e_{2}, \cdots\right.$, $\left.e_{n}\right\}$, suppose $S_{O}$ and $h_{s_{o}}\left(x_{i}\right)$ are DHLTS and DHHFLE, respectively, use them to represent the j-th attribute value of the $\mathrm{i}$-th alternative. The specific procedure of DHHFL-MABAC approach are as follows:

Step 1. Select important attribute indicators to construct a decision matrix $R$ by the DHHFLTS, as shown in the following table.

$$
R=\begin{gathered}
e_{1} \\
c_{2} \\
c_{2} \\
\vdots \\
c_{m}
\end{gathered}\left[\begin{array}{cccc}
a_{11} & a_{12} & \cdots & a_{1 n} \\
a_{21} & a_{22} & \cdots & a_{2 n} \\
\vdots & \vdots & \vdots & \vdots \\
a_{1 m} & a_{2 m} & \cdots & a_{m n}
\end{array}\right]
$$

Next, we use Eq.(9) to get the numeric matrix $\bar{R}$.

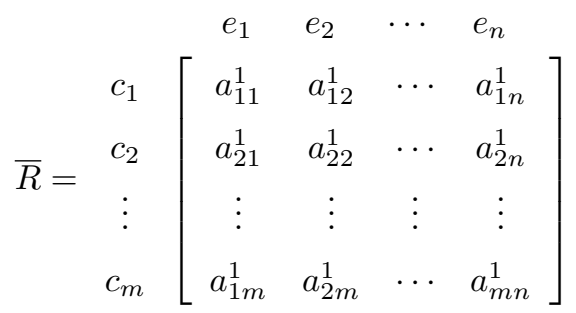

Step 2. Through the DHHFLDM-LCME and the Eq.(22), the initial matrix $(R)$ are normalized.

$$
h_{i j}=\frac{d_{E}\left(a_{i j}, a_{i}^{-}\right)}{d_{E}\left(a_{i}^{+}, a_{i}^{-}\right)}, i=1,2, \cdots, m ; j=1,2, \cdots, n .
$$

Where $h_{i j}$ denotes the evaluation value of the $\mathrm{i}$-th alternative under the $\mathrm{j}$-th attribute after standardization in decision matrix $(R), a_{i}^{+}$represents the DHHFLE corresponding to the maximum value of the $\mathrm{i}$-th alternative under the $\mathrm{j}$-th attribute in the numerical matrix $\bar{R}, a_{i}^{-}$represent the 
DHHFLE correspond with the minimum value of the $\mathrm{i}$-th alternative under the $\mathrm{j}$-th attribute in the numerical matrix $\bar{R}$.

$$
H=\left[\begin{array}{cccc}
h_{11} & h_{12} & \cdots & h_{1 n} \\
h_{21} & h_{22} & \cdots & h_{2 n} \\
\vdots & \vdots & \cdots & \vdots \\
h_{m 1} & h_{m 2} & \cdots & h_{m n}
\end{array}\right]
$$

Step 3. The comprehensive weight $w_{j}$ of attributes is obtained by binary contrast method and entropy weight. $j=1,2, \cdots, n$.

$$
w_{j}=\lambda w_{j}^{1}+(1-\lambda) \frac{1-E_{j}}{\sum_{j=1}^{n}\left(1-E_{j}\right)}
$$

Construct the weighted standardized decision matrix $(\bar{H})$ using the following formula, $w_{j}$ represents the weight of attribute $j$.

$$
\begin{gathered}
h_{i j}^{*}=w_{j}\left(1+h_{i j}\right) \\
\bar{H}=\left[\begin{array}{cccc}
h_{11}^{*} & h_{12}^{*} & \cdots & h_{1 n}^{*} \\
h_{21}^{*} & h_{22}^{*} & \cdots & h_{2 n}^{*} \\
\vdots & \vdots & \cdots & \vdots \\
h_{m 1}^{*} & h_{m 2}^{*} & \cdots & h_{m n}^{*}
\end{array}\right]
\end{gathered}
$$

Step 4. Determine the $B A A$ for each attribute by the Eq.(27), and then we can get the $B A A$ matrix $(G)$.

$$
g_{j}=\left(\prod_{i=1}^{m} h_{i j}^{*}\right)^{\frac{1}{m}}
$$

In which $h_{i j}^{*}$ denotes the elements of weighted standardized decision matrix $(\bar{H}), m$ stands for the total quantity of alternatives.

Step 5. Using the Eq.(28) to calculate the distance to the $B A A$. The distance $(D)$ between the alternative and the $B A A$ is determined as the difference between the element in the weighted matrix $(\bar{H})$ and the value of the $B A A(G)$.

$$
\begin{gathered}
D=\bar{H}-G \\
D=\left[\begin{array}{cccc}
d_{11} & d_{12} & \cdots & d_{1 n} \\
d_{21} & d_{22} & \cdots & d_{2 n} \\
\vdots & \vdots & \cdots & \vdots \\
d_{m 1} & d_{m 2} & \cdots & d_{m n}
\end{array}\right]=\left[\begin{array}{cccc}
h_{11}^{*} & h_{12}^{*} & \cdots & h_{1 n}^{*} \\
h_{21}^{*} & h_{22}^{*} & \cdots & h_{2 n}^{*} \\
\vdots & \vdots & \cdots & \vdots \\
h_{m 1}^{*} & h_{m 2}^{*} & \cdots & h_{m n}^{*}
\end{array}\right]-\left[\begin{array}{cccc}
g_{1} & g_{2} & \cdots & g_{n} \\
g_{1} & g_{2} & \cdots & g_{n} \\
\vdots & \vdots & \cdots & \vdots \\
g_{1} & g_{2} & \cdots & g_{n}
\end{array}\right]
\end{gathered}
$$


where $h_{i j}^{*}$ denotes the weighted matrix of the elements $(\bar{H}), g_{n}$ represents the border approximation area for attribute, $i$ stand for the quantity of alternatives, the quantity of attributes is $j$. In particular, the alternative is closely related to the lower approximation area $(L A A)$, the $B A A$ and the upper approximation area $(U A A)$.

According to the different values of $d_{i j}$, we can get the following situations: The alternatives belong to the $(L A A) G^{-}$when $d_{i j}<0$. The alternatives belong to the $(B A A) G$ when $d_{i j}=0$. The alternative is part of the $U A A\left(G^{+}\right)$when $d_{i j}>0$.

Evidently, the best alternatives must be in the $U A A$ and the worst alternatives must be in the $L A A$.

Step 6. According to the Eq.(30), the overall distance from each alternative to the $B A A$ is calculated, and then sort the alternative according to the value of the distance.

$$
D_{i}=\sum_{j=1}^{n} q_{i j}, \quad i=1,2, \cdots, m
$$




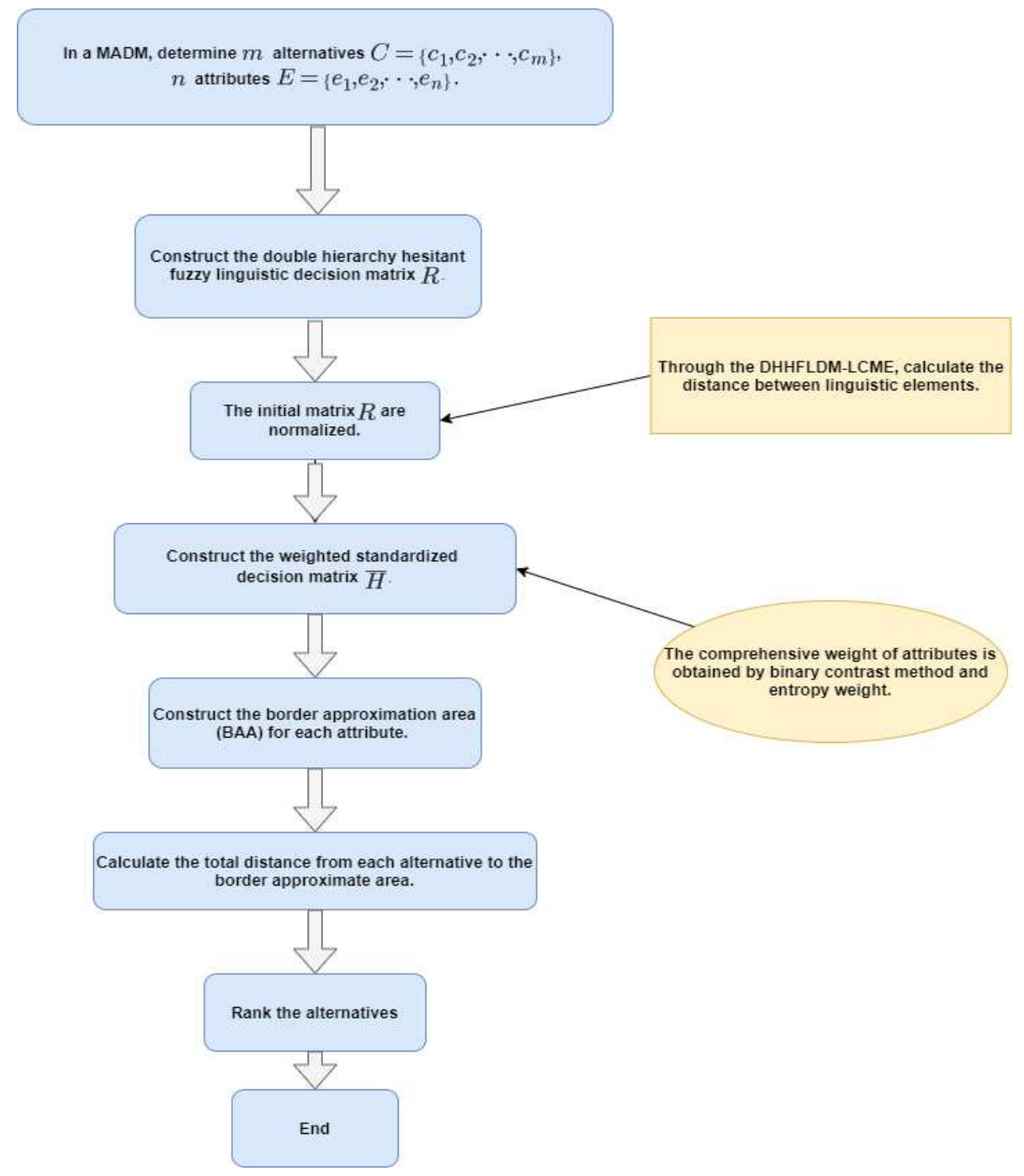

Figure 1: The MADM method of DHHFL-MABAC.

\section{A case study: sewage treament company selection}

\subsection{Background description}

Due to the continuous acceleration of urban construction and development, sewage treatment problem in urban environmental protection has become more and more serious, and the discharge of urban sewage has been increasing, which has caused great harm to water resources and the environment. Therefore, a certain government department aims to solve the problem of sewage treatment. Intends to choose the best one from the four sewage treatment companies for sewage treatment. The evaluated companies include $c_{1}, c_{2}, c_{3}, c_{4}$. The evaluation attributes include four aspects: $e_{1}$ (Achieved water quality standards), $e_{2}$ (Invest funds), $e_{3}$ (Time required), $e_{4}$ (Sewage treatment equipment). The weight is completely unknown.

Let $S=\left\{s_{-3}=\right.$ terrible, $s_{-2}=$ very bad, $s_{-1}=$ bad, $s_{0}=$ medium, $s_{1}=$ good, $s_{2}=$ very good, $s_{3}=$ perfact $\}$ be an LTS for evaluating property performance of the attributes and $O=$ 
$\left\{o_{-3}=\right.$ far from, $o_{-2}=$ little, $o_{-1}=a$ little, $o_{0}=$ ordinary, $o_{1}=$ much, $o_{2}=$ very much, $o_{3}=$ entirely\} be the second hierarchy linguistic term. In this case, the expert evaluated four sewage treatment companies based on four attributes. According to the language expression selected by the expert, the original assessment information can be converted into DHHFLEs.

\subsection{Attribute weight calculation process}

First, we need to figure out the weight of each attribute to prepare for the selection of candidate companies by the DHHFL-MABAC method. The following is the process of weight calculation.

Step 1. Calculate subjective weights based on the binary contrast method, we prioritize the attributes of sewage treatment companies by binary contrast method, the weight of each attribute is obtained. We can get the priority of the attributes: $e_{1} \succ e_{3} \succ e_{2} \succ e_{4}$.

Table 1: The subjective weights matrix

\begin{tabular}{ccccc}
\hline & $e_{1}$ & $e_{2}$ & $e_{3}$ & $e_{4}$ \\
\hline$w_{j}^{1}$ & 0.533 & 0.133 & 0.267 & 0.067 \\
\hline
\end{tabular}

Step 2. Calculate objective weight based on the entropy weight.

First,we can get the normalized matrix from the evaluation matrix $(R)$ in Table 6 .

Table 2: The normalized matrix

\begin{tabular}{ccccc}
\hline & $e_{1}$ & $e_{2}$ & $e_{3}$ & $e_{4}$ \\
\hline$c_{1}$ & 0.377 & 0.603 & 0.000 & 1.000 \\
$c_{2}$ & 1.000 & 0.053 & 0.737 & 0.000 \\
$c_{3}$ & 1.000 & 0.497 & 0.000 & 1.000 \\
$c_{4}$ & 0.335 & 1.000 & 0.000 & 1.000 \\
\hline
\end{tabular}

Next, we can get the information entropy of each attribute.

Table 3: The information entropy matrix

\begin{tabular}{ccccc}
\hline & $e_{1}$ & $e_{2}$ & $e_{3}$ & $e_{4}$ \\
\hline$E_{j}^{2}$ & 0.186 & 0.257 & 0.000 & 0.792 \\
\hline
\end{tabular}

Finally, we can calculate the each attribute's weight through information entropy.

Table 4: The objective weights matrix

\begin{tabular}{ccccc}
\hline & $e_{1}$ & $e_{2}$ & $e_{3}$ & $e_{4}$ \\
\hline$w_{j}^{2}$ & 0.294 & 0.268 & 0.362 & 0.076 \\
\hline
\end{tabular}

Step 3. Calculate the comprehensive weight of the attribute, in this case, $\lambda=0.5$. 
Table 5: The comprehensive weights matrix

\begin{tabular}{ccccc}
\hline & $e_{1}$ & $e_{2}$ & $e_{3}$ & $e_{4}$ \\
\hline$w_{j}$ & 0.414 & 0.201 & 0.315 & 0.720 \\
\hline
\end{tabular}

\subsection{Process based on DHHFL-MABAC method}

In order to prioritize sewage treatment companies and make reasonable selections, next, the flow of the method in this article is introduced.

Step 1. Construct the evaluation matrix $R$ in the form of DHHFLEs.

Table 6: The evaluation matrix $R$

\begin{tabular}{|c|c|c|c|c|}
\hline & $e_{1}$ & $e_{2}$ & $e_{3}$ & $e_{4}$ \\
\hline$c_{1}$ & $\left\{s_{-1<o_{2}>}\right\}$ & $\left\{s_{0<o_{-1}}>\right\}$ & $\left\{s_{-3<o_{0}}>, s_{-2<o_{1}>}, s_{-1<o_{-2}>}\right\}$ & $\left\{s_{1<o_{-2}}>, s_{1<o_{1}>}\right\rangle$ \\
\hline$c_{2}$ & $\left\{s_{1<o_{0}}>, s_{\left.2<o_{0}>s_{3<o_{0}}>\right\}}\right\}$ & $\left\{s_{-1<o_{0}>}\right\}$ & $\left\{s_{1<o_{0}>}, s_{2<o_{-2}>}\right\}$ & $\left\{s_{-1<o_{-2}}>, s_{0<o_{-2}>}\right\}$ \\
\hline$c_{3}$ & $\left\{s_{0<o_{1}}>\right\}$ & $\left\{s_{-1<o_{0}>}, s_{0<o_{2}>}>\right.$ & $\left\{s_{0<o_{-2}}>\right\}$ & $\left\{s_{0<o_{-2}>}, s_{1<o_{-2}>}, s_{2<o_{-2}}>\right\}$ \\
\hline$c_{4}$ & $\left\{s_{0<o_{-3}>}, s_{1<o_{-2}>}\right\}$ & $\left\{s_{1<o_{-2}>}\right\}$ & $\left\{s_{-1<o_{0}>}, s_{0<o_{-1}>}\right\}$ & $\left\{s_{1<o_{-2}>}\right\}$ \\
\hline
\end{tabular}

Next, we use Eq.(9) to get a numeric matrix $\bar{R}$.

Table 7: The numeric matrix $\bar{R}$

\begin{tabular}{ccccc}
\hline & $e_{1}$ & $e_{2}$ & $e_{3}$ & $e_{4}$ \\
\hline$c_{1}$ & 0.444 & 0.444 & 0.222 & 0.639 \\
$c_{2}$ & 0.833 & 0.333 & 0.694 & 0.305 \\
$c_{3}$ & 0.556 & 0.472 & 0.389 & 0.556 \\
$c_{4}$ & 0.445 & 0.556 & 0.389 & 0.556 \\
\hline
\end{tabular}

Step 2. Standardize the elements of the initial matrix $(R)$, and the normalized matrix $(H)$ is obtained.

Table 8: The normalized matrix $H$

\begin{tabular}{ccccc}
\hline & $e_{1}$ & $e_{2}$ & $e_{3}$ & $e_{4}$ \\
\hline$c_{1}$ & 0.000 & 0.498 & 0.000 & 1.000 \\
$c_{2}$ & 1.000 & 0.000 & 1.000 & 0.000 \\
$c_{3}$ & 0.416 & 0.623 & 0.546 & 0.250 \\
$c_{4}$ & 0.208 & 1.000 & 0.546 & 0.250 \\
\hline
\end{tabular}

Step 3. The weighted standardized decision matrix $(\bar{H})$ is constructed. 
Table 9: The weighted standardized decision matrix $H$

\begin{tabular}{ccccc}
\hline & $e_{1}$ & $e_{2}$ & $e_{3}$ & $e_{4}$ \\
\hline$c_{1}$ & 0.413 & 0.301 & 0.314 & 0.143 \\
$c_{2}$ & 0.827 & 0.201 & 0.629 & 0.072 \\
$c_{3}$ & 0.586 & 0.325 & 0.486 & 0.089 \\
$c_{4}$ & 0.499 & 0.401 & 0.486 & 0.089 \\
\hline
\end{tabular}

Step 4. Determining the $B A A$ matrix $(G)$.

Table 10: The $B A A$ matrix

\begin{tabular}{ccccc}
\hline & $e_{1}$ & $e_{2}$ & $e_{3}$ & $e_{4}$ \\
\hline$g_{j}$ & 0.562 & 0.297 & 0.464 & 0.095 \\
\hline
\end{tabular}

Step 5. Calculate the distance to the $B A A(G)$.

Table 11: The distance to the border approximation area

\begin{tabular}{ccccc}
\hline & $e_{1}$ & $e_{2}$ & $e_{3}$ & $e_{4}$ \\
\hline$c_{1}$ & -0.149 & 0.004 & -0.15 & 0.048 \\
$c_{2}$ & 0.265 & -0.096 & 0.165 & -0.023 \\
$c_{3}$ & 0.024 & 0.028 & 0.022 & -0.006 \\
$c_{4}$ & -0.063 & 0.104 & 0.022 & -0.006 \\
\hline
\end{tabular}

Step 6. Rank the alternatives.

Table 12: Rank the alternatives

\begin{tabular}{ccc}
\hline & $D$ & Rank \\
\hline$c_{1}$ & -0.247 & 4 \\
$c_{2}$ & 0.311 & 1 \\
$c_{3}$ & 0.068 & 2 \\
$c_{4}$ & 0.057 & 3 \\
\hline
\end{tabular}

Sorting the alternatives through the DHHFL-MABAC method, we find that the best sewage treatment company is $c_{2}$, and the worst sewage treatment company is $c_{1}$.

\section{Sensitivity analysis}

The sensitivity analysis of the DHHFL-MABAC method has the following three parts. In the first parts, a series of standard weights are given to study the influence of weights on rankings, 
which examines the solutions' stability in the face of weight standard changes. In the second part, sensitivity analysis on the impact of the change of $\lambda$ parameter. The third parts is to compare with other methods and conduct consistency analysis.

\subsection{Sensitivity analysis on the impact of the different weights}

The result of the MADM method depends on a large extent on the weight value of the attribute. Sometimes, when the attribute's weight coefficient varies only little, the final choice will change. Therefore, the sensitivity of MADM results to these changes should usually be analyzed later. Sensitivity analysis is used to determine how changes in attribute weight affect the ranking of alternatives. Table 13 depicts the situations A through E, which correspond to the various weights assigned to each standard and the various priorities assigned to the standard..

Table 13: Situations with different weights of the attributes

\begin{tabular}{lllll}
\hline \multirow{2}{*}{ Situations } & \multicolumn{4}{c}{ Weights of the attributes } \\
\cline { 2 - 5 } & $e_{1}$ & $e_{2}$ & $e_{3}$ & $e_{4}$ \\
\hline A- - equal weights of the attribute & 0.25 & 0.25 & 0.25 & 0.25 \\
$B---$ priority achieved water quality standards & 0.55 & 0.15 & 0.10 & 0.20 \\
$C---$ priority invest funds & 0.15 & 0.55 & 0.20 & 0.10 \\
$D---$ priority time required & 0.10 & 0.15 & 0.50 & 0.15 \\
$E---$ priority achieved water quality standards and invest funds & 0.35 & 0.45 & 0.15 & 0.05 \\
\hline
\end{tabular}

According to the DHHFL-MABAC method, the candidate suppliers with different weight attributes are ranked, the Table 14 manifests the ranking results. Table 15 can more intuitively show the ranking of alternatives in different weight situations. We can see from the table that assigning different weight values to each attribute will lead to different ranking results. In other words, the DHHFL-MABAC mothed is more sensitive to these weights. We compare the optimal solutions in the two situations $\mathrm{B}$ and $\mathrm{D}$ with the results shown in Table 12, and we can see that their optimal solutions are the same. There is not much difference in the sequence of situations between situation B and situation D. The optimal solutions for situations A, C, and E are the same, and the order of the other alternatives is different. This suggests that changing attribute weights may aid in the confirmation of these rankings and consequently the selection of the best option. 
Table 14: Rank the alternatives with different weights of attributes

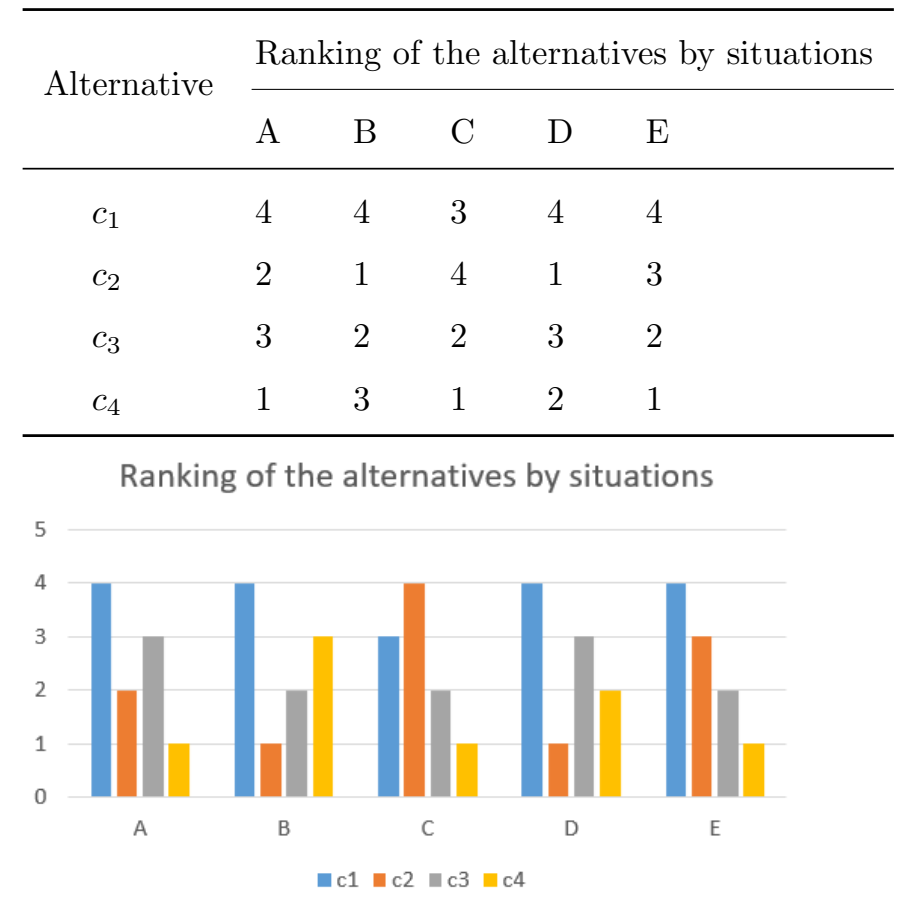

\subsection{Sensitivity analysis on the impact of the change of $\lambda$ parameter}

Through the above analysis of different weight situations, we have obtained that the DHHFLMABAC side is very sensitive to attribute weight values. The comprehensive weight of attributes is obtained by binary contrast method and entropy weight. Through the $\lambda$ parameter, the proportion of subjective weight and objective weight is selected. When the selected subjective weight or objective weight parameter changes very little, the final choice will change. Therefore, we conduct a sensitivity analysis on the impact of the change of $\lambda$ parameter. The following table shows the comprehensive weights under a variety of $\lambda$ parameter values.

Table 15: The comprehensive weights under different values of the $\lambda$ parameter

\begin{tabular}{|c|c|c|c|c|c|c|c|c|c|c|c|c|}
\hline \multirow{2}{*}{ Different values of $\lambda$} & \multicolumn{4}{|c|}{ The objectives weights } & \multicolumn{4}{|c|}{ The subjective weights } & \multicolumn{4}{|c|}{ The comprehensive weight } \\
\hline & $e 1$ & $e 2$ & $e 3$ & $e 4$ & $e 1$ & $e 2$ & $e 3$ & $e 4$ & $e 1$ & $e 2$ & $e 3$ & $e 4$ \\
\hline$\lambda=0.0$ & 0.533 & 0.133 & 0.267 & 0.067 & 0.294 & 0.268 & 0.362 & 0.076 & 0.294 & 0.268 & 0.362 & 0.076 \\
\hline$\lambda=0.1$ & 0.533 & 0.133 & 0.267 & 0.067 & 0.294 & 0.268 & 0.362 & 0.076 & 0.318 & 0.255 & 0.353 & 0.075 \\
\hline$\lambda=0.2$ & 0.533 & 0.133 & 0.267 & 0.067 & 0.294 & 0.268 & 0.362 & 0.076 & 0.342 & 0.241 & 0.343 & 0.074 \\
\hline$\lambda=0.3$ & 0.533 & 0.133 & 0.267 & 0.067 & 0.294 & 0.268 & 0.362 & 0.076 & 0.366 & 0.228 & 0.334 & 0.073 \\
\hline$\lambda=0.4$ & 0.533 & 0.133 & 0.267 & 0.067 & 0.294 & 0.268 & 0.362 & 0.076 & 0.390 & 0.214 & 0.324 & 0.072 \\
\hline$\lambda=0.5$ & 0.533 & 0.133 & 0.267 & 0.067 & 0.294 & 0.268 & 0.362 & 0.076 & 0.414 & 0.201 & 0.315 & 0.071 \\
\hline$\lambda=0.6$ & 0.533 & 0.133 & 0.267 & 0.067 & 0.294 & 0.268 & 0.362 & 0.076 & 0.437 & 0.187 & 0.305 & 0.071 \\
\hline$\lambda=0.7$ & 0.533 & 0.133 & 0.267 & 0.067 & 0.294 & 0.268 & 0.362 & 0.076 & 0.461 & 0.174 & 0.300 & 0.070 \\
\hline$\lambda=0.8$ & 0.533 & 0.133 & 0.267 & 0.067 & 0.294 & 0.268 & 0.362 & 0.076 & 0.485 & 0.160 & 0.286 & 0.069 \\
\hline$\lambda=0.9$ & 0.533 & 0.133 & 0.267 & 0.067 & 0.294 & 0.268 & 0.362 & 0.076 & 0.509 & 0.147 & 0.277 & 0.068 \\
\hline$\lambda=1.0$ & 0.533 & 0.133 & 0.267 & 0.067 & 0.294 & 0.268 & 0.362 & 0.076 & 0.533 & 0.133 & 0.267 & 0.067 \\
\hline
\end{tabular}




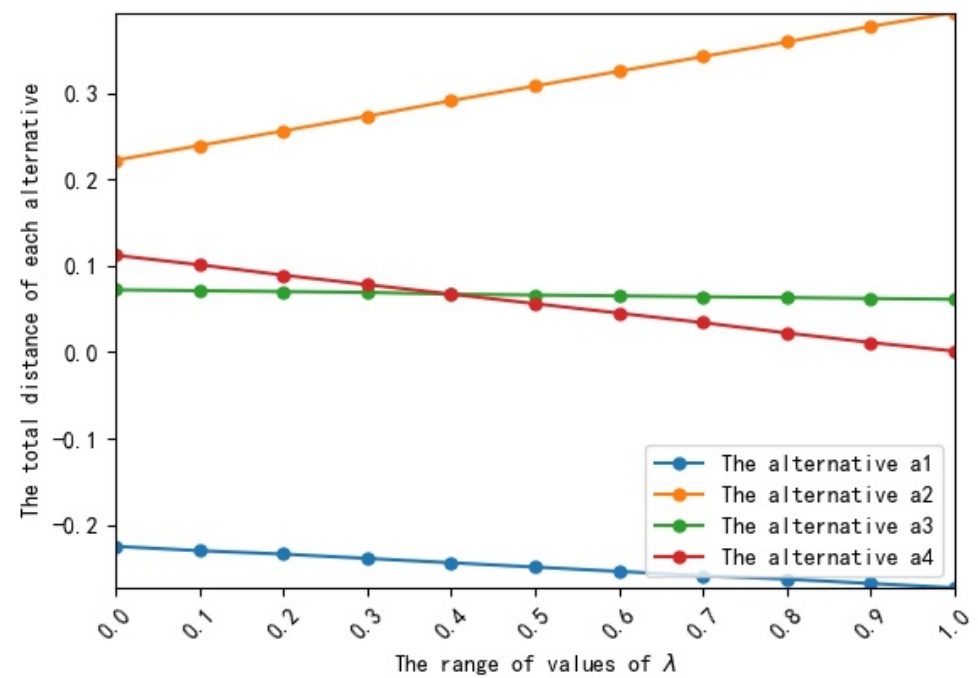

Figure 2: The total ditance of each alternative under different values of the $\lambda$ parameter.

From the above figure, we can see that with the continuous change of the value of $\lambda$, the comprehensive weight of attributes changes continuously. When the value of $\lambda$ increases, the comprehensive weight of attribute $e_{1}$ increases and the comprehensive weight of attribute $e_{2}, e_{3}$ and $e_{4}$ decreases. As the value of $\lambda$ changes, the total distance of each alternative also changes. Among them, the total distance of $c_{1}$ and $c_{4}$ decreases, while the total distance of $c_{2}$ and $c_{3}$ increases. This shows that different a values will change the comprehensive weight of attributes and affect the total distance of each alternative.

\subsection{Compare with MULTIMOORA method and conduct consistency analysis}

MULTIMOORA is a reasonable and effective multi-objective optimization method. Gou 35] extended the DHHFL-MULTIMOORA method based on the traditional MULTIMOORA method. The DHHFL-MULTIMOORA method takes the comprehensive consideration of the DHHFLRS, the DHHFLRP and the DHHFLFMF measures, and finally determines the most excellent plan. Next, we work out the problem of selection of sewage treatment companies by using the DHHFLMULTIMOORA method, and compare it with the DHHFL-MABAC method to prove the improvement and advantages of the DHHFL-MABAC method.

Step 1. Convert the original evaluation information into a numeric matrix, shown in Table 16. 
Table 17: The normalization results of all DHHFLEs

\begin{tabular}{lllll}
\hline & $e_{1}$ & $e_{2}$ & $e_{3}$ & $e_{4}$ \\
\hline$c_{1}$ & 0.195 & 0.246 & 0.131 & 0.311 \\
$c_{2}$ & 0.366 & 0.184 & 0.450 & 0.148 \\
$c_{3}$ & 0.244 & 0.261 & 0.230 & 0.270 \\
$c_{4}$ & 0.195 & 0.308 & 0.230 & 0.270 \\
\hline
\end{tabular}

Table 16: The numeric matrix $\bar{R}$

\begin{tabular}{lcllc}
\hline & $e_{1}$ & $e_{2}$ & $e_{3}$ & $e_{4}$ \\
\hline$c_{1}$ & 0.444 & 0.444 & 0.222 & 0.639 \\
$c_{2}$ & 0.833 & 0.333 & 0.694 & 0.305 \\
$c_{3}$ & 0.556 & 0.472 & 0.389 & 0.556 \\
$c_{4}$ & 0.445 & 0.556 & 0.389 & 0.556 \\
\hline
\end{tabular}

Step 2. Use DHHFLRS to sort the alternatives.

$$
h_{s_{o_{i j}}}^{o}=E\left(h_{s_{o_{i j}}}\right) / \sum_{i=1}^{m} E\left(h_{s_{o_{i j}}}\right)
$$

The numerical matrix is normalized according to Eq.(31) and Eq.(9), Table 17 shows the outcomes. In addition, we can calculate the aggregate ratio $\Phi_{i}$ for each alternative by Eq.(32), shown in Table 18.

$$
\Phi_{i}=\sum_{j=1}^{\theta} h_{s_{o_{i j}}}^{o}-\sum_{j=\theta+1}^{m} h_{s_{o_{i j}}}^{o}
$$

In the case of this paper, consider that all attributes are indicators of profitability, where $\theta$ represents the number of benefit attribute and $m-\theta$ is the quantity of cost attribute. Thus $\Phi_{i}$ stands for the best performance value of i-th alternative.

Table 18: The final summarizing ratios and the rank of alternative

\begin{tabular}{lcccc}
\hline & $c_{1}$ & $c_{2}$ & $c_{3}$ & $c_{4}$ \\
\hline$\Phi_{i}$ & 0.883 & 1.148 & 1.005 & 1.003 \\
rank & 4 & 1 & 2 & 3 \\
\hline
\end{tabular}

Step 3. The alternatives are ranked by using DHHFLRP. We calculate maximal objective reference points $M_{j}(i=1,2,3,4 ; j=1,2,3,4)$ by Eq.(33). Table 19 shows the results.

$$
M_{j}=\left\{\begin{array}{l}
\max _{i}\left\{h_{s_{o_{i j}}}^{o}\right\}, \text { if } j \leq \theta \\
\min _{i}\left\{h_{s_{o_{i j}}}^{o}\right\}, \text { if } j>\theta
\end{array}\right.
$$


Table 19: The maximal objective reference point $M_{j}$ of each attribute

\begin{tabular}{cccc}
\hline$M_{1}$ & $M_{2}$ & $M_{3}$ & $M_{4}$ \\
\hline$\left\{s_{1<o_{0}>}, s_{2<o_{0}>} s_{3<o_{0}>}\right\}$ & $\left\{s_{1<o_{-2}>}\right\}$ & $\left\{s_{1<o_{0}>}, s_{2<o_{-2}}\right\rangle$ & $\left\{s_{1<o_{-2}>,}, s_{1<o_{1}>}\right\}$ \\
\hline
\end{tabular}

Next, according to the Eq.(34), the distance between each DHHFLE $h_{s_{o_{i j}}}^{o}$ and $M_{j}$ is calculated. The calculation result was as shown in Table 20.

$$
D\left(h_{s_{o_{i j}}}^{o}, M_{j}\right)=\sqrt{\frac{1}{L} \sum_{l=1}^{L}\left(\omega_{1}^{l}-\omega_{2}^{l}\right)^{2}} \quad\left(\omega_{1}^{l} \in F\left(h_{s_{o_{i j}}}^{o}\right), \omega_{2}^{l} \in F\left(M_{j}\right)\right)
$$

Table 20: The distance between each DHHFLE $h_{s_{o_{i j}}}^{o}$ and $M_{j}$ and the final ranking

\begin{tabular}{ccccccc}
\hline & $e_{1}$ & $e_{2}$ & $e_{3}$ & $e_{4}$ & $\max _{j}\left\{D\left(h_{s_{o_{i j}}}^{o}, M_{j}\right)\right\}$ & Ranking \\
\hline$c_{1}$ & 0.412 & 0.111 & 0.553 & 0.000 & 0.553 & 3 \\
$c_{2}$ & 0.000 & 0.222 & 0.000 & 0.333 & 0.333 & 2 \\
$c_{3}$ & 0.309 & 0.162 & 0.307 & 0.118 & 0.309 & 1 \\
$c_{4}$ & 0.561 & 0.000 & 0.307 & 0.118 & 0.561 & 4 \\
\hline
\end{tabular}

Step 4. The alternatives are ranked by using DHHFLFMF. The calculation results are shown in Table 21.

$$
U_{i}=\Im_{i} \otimes \aleph_{i}=\prod_{j=1}^{\theta} E\left(h_{s_{o_{i j}}}^{o}\right)
$$

Table 21: The overall expected values of all alternatives and the rank of alternatives

\begin{tabular}{lcccc}
\hline & $c_{1}$ & $c_{2}$ & $c_{3}$ & $c_{4}$ \\
\hline$U_{i}$ & 0.028 & 0.059 & 0.057 & 0.054 \\
rank & 4 & 1 & 2 & 3 \\
\hline
\end{tabular}

Step 5. We finally obtained the ranking of alternatives by calculating and comparing the results of DHHFLRS, DHHFLRP and DHHFLFMF.

Table 22: The normalization results of all DHHFLEs

\begin{tabular}{ccccc}
\hline & $D H H F L R S$ & $D H H F L R P$ & $D H H F L F M F$ & $D H H F L-M U L T I M O O R A$ \\
\hline$c_{1}$ & 4 & 3 & 4 & 4 \\
$c_{2}$ & 1 & 2 & 1 & 1 \\
$c_{3}$ & 2 & 1 & 2 & 2 \\
$c_{4}$ & 3 & 4 & 3 & 3 \\
\hline
\end{tabular}


Table 23: Comparison of alternatives sorting results

\begin{tabular}{lcccc}
\hline & $c_{1}$ & $c_{2}$ & $c_{3}$ & $c_{4}$ \\
\hline DHHFL $-M A B A C(\lambda=0.1)$ & 4 & 1 & 3 & 2 \\
DHHFL $-M A B A C(\lambda=0.3)$ & 4 & 1 & 3 & 2 \\
DHHFL - MABAC $(\lambda=0.5)$ & 4 & 1 & 2 & 3 \\
DHHFL - MABAC $(\lambda=0.7)$ & 4 & 1 & 2 & 3 \\
DHHFL - MABAC $(\lambda=0.9)$ & 4 & 1 & 2 & 3 \\
DHHFL - MULTIMOORA & 4 & 1 & 2 & 3 \\
\hline
\end{tabular}

By comparing with the DHHFL-MULTIMOORA method, the optimal alternative obtained by the two methods are consistent, which also illustrates the feasibility of the DHHFL-MABAC method. What's more, the DHHFL-MABAC method has more advantages in some aspects. The DHHFL-MABAC method can get a strong ranking, while the DHHFL-MULTIMOORA method cannot accurately rank the alternatives. If the DHHFL-MULTIMOORA method is adopted, the results of the three measures may be completely different, so that the comprehensive ranking cannot be obtained. The DHHFL-MABAC method sorts the alternatives by calculating the global distance of each alternative, and its calculation process is simple and efficient. Compared with the traditional method, the DHHFL-MABAC decision matrix contains more comprehensive and detailed evaluation information. The DHHFL-MABAC method considers potential losses in the calculation process, and also considers the preference information of decision-makers and the importance of attributes, making the calculation results more accurate and reasonable. When the parameter $\lambda$ takes different values, the DHHFL-MABAC method sorts the alternatives differently. By setting the value of $\lambda$, a more reasonable order of the alternatives can be obtained.

\section{Conclusion}

This paper proposes the DHHFL-MABAC method based on distance measure and comprehensive weight. To this end, we first define a double hierarchy hesitant fuzzy linguistic distance measure based on the least common multiple to calculate the difference between DHHFLEs, and detailed research its properties. Next, we linearly combine the proposed binary contrast method with entropy weight to get the comprehensive weight, which not only considers the effectiveness of objective weights, but also considers the preference information of decision makers. This method can make the determined weight more effective and reasonable. Then, we apply the DHHFL-MABAC method to the MADM problem chosen by the sewage treatment company. Finally, a sensitivity analysis is performed on the DHHFL-MABAC method. The sensitivity analysis includes three aspects. The first is to sensitivity analysis on the impact of the change of $\lambda$ parameter. The second is to detect the impact on the ranking of alternatives by giving different weight values to. The third is to compare the results with other MADM methods to analyze the consistency of the results. The research results of this paper prove that this method is an effective and useful tool for decision-making. 
The research on determining attribute weights is very meaningful in the future application of DHHFL-MABAC method. In addition, we can also extend the DHHFL-MABAC method to work out the problem of multi-attribute group decision making.

\section{Acknowledgment}

This work was supported by the Graduate Teaching Reform Research Program of Chongqing Municipal Education Commission (No.YJG212022) and Chongqing Research and Innovation Project of Graduate Students(No.CYS21326).

\section{Declarations}

1. Articles do not relying on clinical trials.

2. The authors declare that they have no conflict of interest.

3. All submitted manuscripts containing research which does not involves human participants and/or animal experimentation.

\section{References}

[1] Zadeh L A. (1965). Fuzzy set. Information and Control, 8(3), 338-353.

[2] Zadeh L A. (1975). The concept of a linguistic variable and its application to approximate reasoning. Information Sciences, 8(3), 199-249.

[3] Goguen. (1967). L-fuzzy sets, Journal of Mathematic Analysis and Application, 20(1), 145-174.

[4] Turksen. (1986). Interval-fuzzy sets, Fuzzy Sets and Systems, 20, 191-210.

[5] Herrera F, Martinez L. (2000). A 2tuple fuzzy linguistic representation model for computing with words, IEEE Transactions on Fuzzy Systems, 8(6), 746-752.

[6] Atanassov K, Stoeva S. (1986). Intuitionistic fuzzy sets, Fuzzy Sets and Systems, 20(1), 87-96.

[7] Atanassov K, Gargov G. (1989). Interval valued intuitionistic fuzzy sets, Fuzzy Sets and Systems, 31(3), 343-349.

[8] Torra V. (2010). Hesitant fuzzy sets, International Journal Of Intelligent Systems, 25(6), 529539.

[9] Wu P, Zhou L. (2020). Muli-stage optimization model for hesitant qualitative decision making with hesitant furzy linguistic preference relations, Applied Intelligence, 50(1), 222-240. 
[10] Wu P, J Zhu, Zhou L. (2019). Automatic iterative algorithm with local revised strategies to improve the consistency of hesitant fuzzy linguistic preference relations, International Journal of Fuzzy Systems, 21(7), 2283-2298.

[11] Wang H. (2015). Extended hesitant fuzzy linguistic term sets and their aggregation in group decision making, International Journal of Computational Intelligence Systems, 8(1), 14-33.

[12] I Beg, Tabasam Rashid. (2013). Topsis for hesitant fuzzy linguistic term sets, International Journal of Intelligent Systems, 28(12), 1162-1171.

[13] I Beg, Tabasam Rashid. Group decision making using comparative linguistic expression based on hesitant intuitionistic fuzzy sets, Applications and Applied Mathematics, 10(2), 1082-1092.

[14] Gou X, Liao H, Xu Z. (2017). Double hierarchy hesitant fuzzy linguistic term set and multimoora method: a case of study to evaluate the implementation status of haze controlling measures, Information Fusion, 38(3), 22-34.

[15] Gou X, Xu Z, Liao H. (2018). Multiple criteria decision making based on distance and similarity measures under double hierarchy hesitant fuzzy linguistic environment, Computers and Industrial Engineering, 126, 516-530.

[16] Gou X, Xu Z, Zhou W. (2020). Managing consensus by multi-stage optimization models with linguistic preference orderings and double hierarchy linguistic preferences, Technological and Economic Development of Economy, 26(3), 1-33.

[17] Krishankumar R, Subrajaa L S, Ravichandran K S. (2019). A framework for multi-attribute group decision-making using double hierarchy hesitant fuzzy linguistic term set, International Journal of Fuzzy Systems, 21(4), 1130-1143.

[18] Krishankumar R, Ravichandran K S, Shyam V. (2020). Multi-attribute group decision-making using double hierarchy hesitant fuzzy linguistic preference information, Neural Computing and Applications, 32(17), 14031-14045.

[19] X Liu, X Wang, Qu Q. (2018). Double hierarchy hesitant fuzzy linguistic mathematical programming method for MAGDM based on shapley values and incomplete preference information, IEEE Journal of Translational Engineering in Health and Medicine, 6, 74162-74179.

[20] Liu N, He Y, Xu Z. (2019). Evaluate public-private-partnerships advancement using double hierarchy hesitant fuzzy linguistic promethee with subjective and objective information from stakeholder perspective, Technological and Economic Development of Economy, 25(3), 1-35.

[21] Krishankumar R, Ravichandran K S, Kar S. (2021). Double hierarchy hesitant fuzzy linguistic term set-based decision framework for multi-attribute group decision-making, Soft Computing, $25(7), 1-21$. 
[22] Zhang R, Xu Z, Gou X. (2021). An integrated method for multi-criteria decision-making based on the best-worst method and Dempster-Shafer evidence theory under double hierarchy hesitant fuzzy linguistic environment. Applied Intelligence, 51(2), 713-735.

[23] Liu P, Shen M, Teng F. (2020). Double hierarchy hesitant fuzzy linguistic entropy-based TODIM approach using evidential theory, Information Sciences, 547(1), 223-243.

[24] Wang X, Gou X, Xu Z. (2019). Assessment of traffic congestion with ORESTE method under double hierarchy hesitant fuzzy linguistic environment. Applied Soft Computing, 86, 1-13.

[25] R.M. Rodriguez, L.Martnez, Torra V. (2014). Hesitant fuzzy sets: state of the art and future directions. International Journal of Intelligent Systems, 29(6), 495-524.

[26] Liao H, Xu Z, Zeng X J. (2014). Distance and similarity measures for hesitant fuzzy linguistic term sets and their application in multi-criteria decision making. Information Sciences, 271, $125-142$.

[27] Gao J, Xu Z, Liao H. (2017). A dynamic reference point method for emergency response under hesitant probabilistic fuzzy environment. International Journal of Fuzzy Systems, 19(5), 1261-1278.

[28] Peng W, Zhou L, H Chen. (2019). Additive consistency of hesitant fuzzy linguistic preference relation with a new expansion principle for hesitant fuzzy linguistic term sets. IEEE Transactions on Fuzzy Systems, 27, 716-730.

[29] Shi Y T, Jie L, Peng W. (2017). Application of entropy method in river health evaluation based on aquatic ecological function regionalization. IOP Conference Series: Earth and Environmental Science, 63, 12-46.

[30] Wang X, Zhou Z, Sun L. (2021). Study on the whole process evaluation of new energy grid connection based on AHP-entropy weight method. IOP Conference Series: Earth and Environmental Science, 831, 12-23.

[31] Verma R. (2021). Fuzzy MABAC method based on new exponential fuzzy information measures. Soft Computing, 25(14), 9575-9589.

[32] Pamucar D, Cirovic G. (2015). The selection of transport and handling resources in logistics centers using multi-attributive border approximation area comparison (mabac). Expert Systems with Applications, 42(6), 3016-3028.

[33] Wang P, Wang J. (2019). The multi-attributive border approximation area comparison (mabac) for multiple attribute group decision making under 2-Tuple linguistic neutrosophic environment. Informatica, 30(4), 799-818. 
[34] Dai, Jingguo, Peng. (2017). Hesitant fuzzy soft decision making methods based on WASPAS, MABAC and COPRAS with combined weights. Journal of Intelligent and Fuzzy Systems, 33(2), $1-13$.

[35] Gou X, Liao H, Xu Z. (2017). Double hierarchy hesitant fuzzy linguistic term set and MULTIMOORA method: a case of study to evaluate the implementation status of haze controlling measures. Information Fusion, 38, 22-34. 